\title{
Hormones and Hearing: Central Auditory Processing in Women
}

DOI: $10.3766 /$ jaaa.17123

\author{
Skylar Trott* \\ Trey Cline $\dagger$ \\ Jeffrey Weihing \\ Deidra Beshear§ \\ Matthew Bush $\dagger$ \\ Jennifer Shinn $\dagger$
}

\begin{abstract}
Background: Estrogen has been identified as playing a key role in many organ systems. Recently, estrogen has been found to be produced in the human brain and is believed contribute to central auditory processing. After menopause, a low estrogen state, many women report hearing loss but demonstrate no deficits in peripheral hearing sensitivity, which support the notion that estrogen plays an effect on central auditory processing. Although animal research on estrogen and hearing loss is extensive, there is little in the literature on the human model.
\end{abstract}

Purpose: The aim of this study was to evaluate relationships between hormonal changes and hearing as it relates to higher auditory function in pre- and postmenopausal (Post-M) females.

Research Design: A prospective, group comparison study.

Study Sample: Twenty eight women between the ages of 18 and 70 at the University of Kentucky were recruited.

Data Collection and Analysis: Participants were separated into premenopausal and peri-/Post-M groups. Participants had normal peripheral hearing sensitivity and underwent a behavioral auditory processing battery and electrophysiological evaluation. An analysis of variance was performed to address the aims of the study.

Results: Results from the study demonstrated statistically significant difference between groups, where Post-M females had difficulties in spatial hearing abilities as reflected on the Listening in Spatialized Noise Test-Sentences test. In addition, measures on the auditory brainstem response and the middle latency response reflected statistically significant differences between groups with Post-M females having longer latencies.

Conclusions: Results from the present study demonstrated significant differences between groups, particularly listening in noise. Females who present with auditory complaints in spite of normal hearing thresholds should have a more extensive audiological evaluation to further evaluate possible central deficits.

Key Words: central auditory processing disorder, menopause

Abbreviations: $\mathrm{ABR}=$ auditory brainstem response; $\mathrm{ANOVA}=$ analysis of variance; CAPD $=$ central auditory processing disorder; DD = dichotic digits; HRT = hormone replacement therapy; ILD = interaural latency difference; LiSN-S = Listening in Spatialized Noise Test-Sentences; MLR = middle latency response; Peri-M = perimenopausal; Post-M = postmenopausal; Pre-M = premenopausal; SPIN-R = Speech Perception in Noise Test Revised; SRT = speech recognition threshold

*University of Kentucky College of Medicine, Lexington, KY; 'Department of Otolaryngology-Head and Neck Surgery, University of Kentucky, Lexington, KY; "Department of Audiology, University of Louisville, Louisville, KY; §Divisions of Women's Health, Department of Internal Medicine, University of Kentucky, Lexington, KY

Corresponding author: Jennifer Shinn, Department of Otolaryngology-Head and Neck Surgery, University of Kentucky, Lexington, KY 40536; Email: jennifer.shinn@uky.edu 


\section{INTRODUCTION}

$\mathrm{E}$ strogen has been found to play an integral role in many organ systems including the auditory system. The auditory system comprises a peripheral component, which generally includes the outer, inner, and middle ear, and a central component, which consists of structures within the central nervous system. In this auditory system, estrogen receptors have been shown to be present in the pathways of mice and rats, which suggests that estrogen may play an important role in hearing (Stenberg et al, 1999). It has been demonstrated in rats that ovariectomy negatively affects auditory brainstem responses (ABR) and middle latency responses (MLR) (Coleman et al, 1994). Estrogen replacement therapy may reverse the neurophysiological changes in these rats following ovariectomy (Coleman et al, 1994). In songbirds such as the white-throated sparrow, auditory processing has been shown to be dependent on levels of estradiol, which subsequently affects females' behavioral responses to male songs during breeding season (Maney et al, 2006). In female rhesus monkeys, administration of exogenous estrogen resulted in shorter ABR latencies (Golub et al, 2004).

In humans, estrogen receptors have been identified in the inner ear (Stenberg et al, 2001). Estrogen has been found to be produced in the human brain by auditory neurons themselves and has an important direct effect on neurotransmission (Pinaud and Tremere, 2012). Estrogen has an effect on neurotransmission by acting as a neuromodulator thereby directly influencing synaptic physiology by suppressing presynaptic $\gamma$-aminobutyric acid (GABA) release - in addition to estrogen-dependent genomic transcription in auditory neurons (Pinaud and Tremere, 2012). Although less direct, estrogen has also been shown to influence cochlear blood flow, which may play a role in auditory function (Laugel et al, 1987).

Because of estrogen's role in the auditory system, postmenopausal (Post-M) women may suffer auditory processing disorders due to decreased production of the hormone. Women consistently have shorter latencies and larger amplitudes on ABR compared with men between the ages of 20 and 79 (Jerger and Hall, 1980). However, older Post-M women have similar latencies as men of the same age (Wharton and Church, 1990). Thus, female ABR latencies, which are normally shorter compared with men, may prolong after menopause to the point that female latencies are similar to male latencies (Wharton and Church, 1990). Administration of hormone replacement therapy (HRT) in Post-M women has been shown to improve ABR latencies (Caruso et al, 2000; Caruso et al, 2003; Khaliq et al, 2003). Turner's syndrome, an estrogen deficient state, is associated with earlier onset of high-frequency hearing loss in addition to other auditory problems associated with the condition, such as recurrent otitis media, sen- sorineural hearing loss, conductive hearing loss, or inner ear malformations (Hultcrantz and Sylven, 1997; Hultcrantz et al, 2006). In addition, it has been shown that estradiol therapy may delay hearing loss in Post-M women (Kilicdag et al, 2004). These findings support the notion that the hormonal changes accompanying menopause have an effect on hearing.

Hearing loss is an exceedingly common disorder in the United States. As many as $20 \%$ of Americans aged 12 years and older have hearing loss and the prevalence increases with every decade of age (Lin et al, 2011). Although hearing loss is not associated with mortality, this condition substantially affects the quality of life of elderly individuals. Hearing loss in the elderly has been associated with dysfunction in emotional states, social interactions, and communications and many consider these dysfunctions to be severely handicapping (Mulrow et al, 1990a,b). Although the focus of hearing loss has been primarily on peripheral involvement, central deficits are less commonly studied but can negatively influence quality of life as well.

Many Post-M women report abnormal auditory perceptions, complaining of difficulty in hearing or tinnitus but demonstrate no deficits in peripheral hearing sensitivity as reflected on the pure-tone audiogram. It has been shown that participants with these auditory complaints but normal audiometry may have an underlying central auditory processing disorder (CAPD) (Shinn et al, 2016). Although there is extensive research examining estrogen and hearing loss using animal models, there is little research examining these relationships between humans and auditory processing. The aim of this study was to evaluate relationships between hormonal changes and hearing as it relates to higher auditory function in premenopausal (Pre-M) and Post-M females.

\section{MATERIALS AND METHODS}

\section{Participants}

A prospective, group comparison study was conducted on 28 adult women between the ages of 18 and 70 at the University of Kentucky between September 2015 and April 2017. This study was approved by the University of Kentucky Institutional Review Board (IRB \#150623-P2H). Participants were separated into Pre-M and Peri/Post-M groups. There were 14 participants in each group, respectively. Perimenopausal participants were grouped with Post-M participants due to lack of perimenopausal participants enrolling in the study (a total of three were enrolled). Participants were recruited via flyers and word of mouth. The average age of the Pre-M group was 30 years old and the average age of the Peri/Post-M group was 54 years old. Pre-M was defined as having regular cycles, 
Table 1. Audiogram and Word Recognition Results

\begin{tabular}{|c|c|c|c|c|c|c|}
\hline \multirow[b]{2}{*}{ Group } & \multirow[b]{2}{*}{ Ear } & \multicolumn{4}{|c|}{ Mean Pure-Tone Thresholds (dB) } & \multirow[b]{2}{*}{ WR (\%) } \\
\hline & & $500 \mathrm{~Hz}$ & $1000 \mathrm{~Hz}$ & $2000 \mathrm{~Hz}$ & $4000 \mathrm{~Hz}$ & \\
\hline \multirow[t]{2}{*}{ Pre-M } & Left & 3.9 & 4.3 & 3.9 & 6.4 & 98.0 \\
\hline & Right & 5 & 5 & 6.1 & 7.5 & 99.4 \\
\hline \multirow[t]{2}{*}{ Post-M } & Left & 7.5 & 9.3 & 6.8 & 12.9 & 98.9 \\
\hline & Right & 6.8 & 8.6 & 7.1 & 12.1 & 98.3 \\
\hline
\end{tabular}

$\overline{\mathrm{WR}}=$ mean word recognition percent correct.

perimenopausal was defined as having irregular cycles between three and 11 months, and Post-M was defined as having at least one year of amenorrhea. Inclusion criteria were defined as normal hearing (pure-tone audiological thresholds better than $25 \mathrm{~dB}$ HL at 500, 1000, 2000, and $4000 \mathrm{~Hz}$ ), right handed, and no active otologic or neurologic involvement. The audiogram and word recognition results are depicted in Table 1 for each group. Participants were excluded if they had any of the following characteristics: left-handedness, hearing loss, history of neurologic involvement per participant report, active otologic involvement per participant report. Additional exclusionary criteria included actively taking glucocorticoids, pregnant or suspicion of pregnancy, history of hysterectomy, use of HRT within the past three months, use of birth control within the past three months, or use of estrogen-based medications for other conditions.

\section{Procedures}

\section{Behavioral Measures}

All behavioral testing was completed in a soundtreated suite. Each participant underwent a comprehensive audiological evaluation. A traditional audiological evaluation including pure-tone air conduction and speech audiometry was completed using a GSI AudioStar Pro (Grason-Stadler, Eden Prairie, MN). The behavioral auditory processing battery consisting of dichotic digits (DD) test, duration patterns test, Listening in Spatialized Noise Test-Sentences (LiSN-S) test, and the Speech Perception In Noise-Revised (SPIN-R) test. Test stimuli were administered on an iPad and passed through a diagnostic audiometer to supra-aural headphones earphones. The LiSN-S is administered via computer and stimuli are delivered through Sennheiser HD 215 headphones (Sennheiser, Old Lyme, CT). The stimuli were presented at $50-\mathrm{dB}$ sensation level re: pure-tone average to each ear. All participants were given practice items, which were placed at the beginning of the test to insure the task was understood. Participants were asked to verbally repeat what they heard and the tests were manually scored by the examiner (DD, duration patterns, and SPIN) or by the computer program (LiSN). The DD test is a well-established test of binaural integration (Musiek, 1983). This is a low linguistic measure involv- ing the simultaneous presentation of two numbers to each ear (four in total) and participants were asked to repeat all four numbers. Percent correct scores were recorded. The duration pattern test was selected to further evaluate participants' temporal processing ability with respect to ordering using a low linguistic measure (Musiek et al, 1990). Participants were presented with stimuli that are either long or short in duration and asked to repeat the pattern. This test was administered in the sound field and was scored as a percent correct. The R-SPIN evaluates participant ability to process auditory information in the presence of background noise (multitalker babble with $\mathrm{a}+8 \mathrm{~dB}$ signal-to-noise ratio) (Bilger et al, 1984). The R-SPIN presents participants with 50 sentences per list that are either high or low predictability. Participants were evaluated in the sound field and scores were calculated for both the low and high predictability sentences as a percent correct score. The LiSN-S is a clinical measure which uses virtual reality to measure speech perception in noisy environments (Cameron and Dillon, 2007). The LiSN-S was administered in four test conditions: same speaker with masker at $0^{\circ}$ azimuth, same speaker $\pm 90^{\circ}$ azimuth,

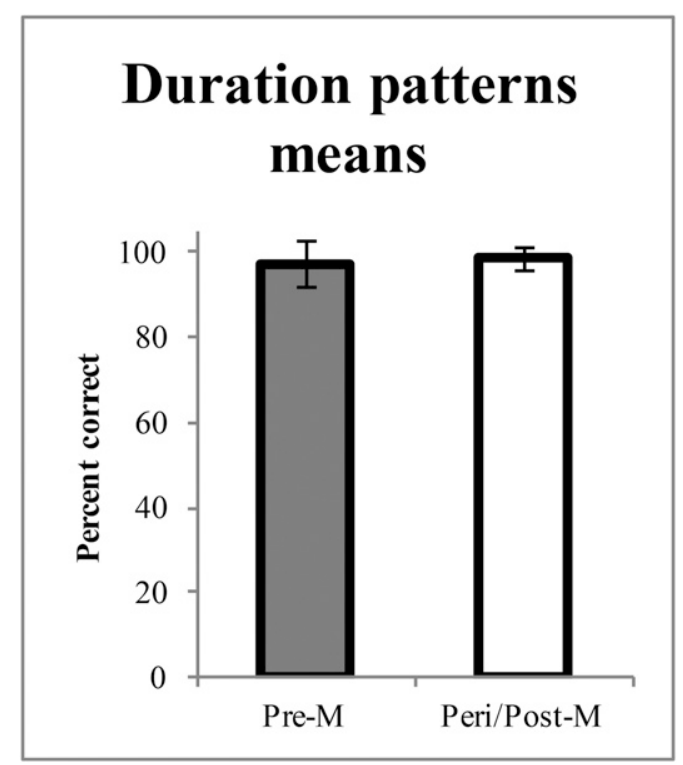

Figure 1. Means for the duration pattern test for pre- vs. postmenopausal women. Error bars represent one standard deviation. Pre-M = premenopausal; Peri/Post-M = peri-/postmenopausal. 


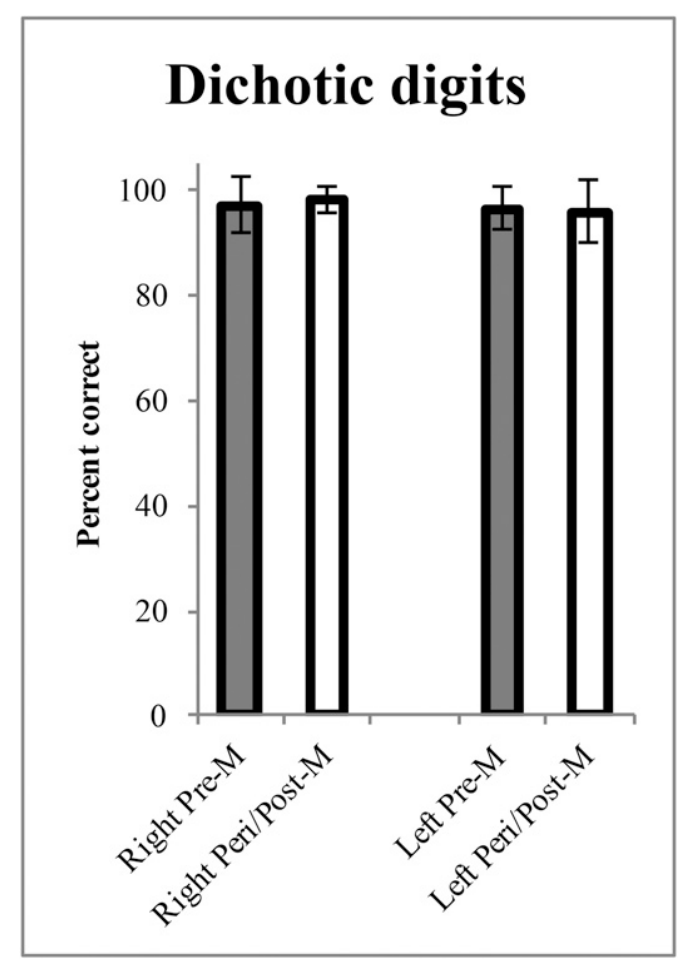

Figure 2. Means for the dichotic digits test for pre- vs. postmenopausal women between ears. Error bars represent one standard deviation. Pre-M = premenopausal; Peri/Post-M = peri-/postmenopausal.

different speaker $0^{\circ}$ azimuth, and different speaker \pm $90^{\circ}$ azimuth. The LiSN-S is scored as the participant's speech reception threshold. The order of behavioral testing was pseudorandomized with standardized instructions read to participants before each test.

\section{Electrophysiological Measures}

In addition, participants underwent an electrophysiological evaluation consisting of $\mathrm{ABR}$ and MLR using the Bio-logic ${ }^{\circledR}$ Navigator $^{\circledR}$ Pro. Both the ABR and MLR measure electrical potentials generated by a stimulus as they travel through the central auditory nervous system, with each wave representing major generators along the pathway. Electrode montages were identical for both the ABR and MLR. For each participant, the electrode sites (bilateral mastoid processes and forehead) were prepped before placement of the electrodes. Impedances were $<3 \mathrm{k} \Omega$ across the electrode array before the start of the testing.

The ABR was recorded using a $100-\mu$ sec rarefaction click stimulus at $80 \mathrm{~dB} \mathrm{nHL}$. The ABR latencies were collected for waves I, III, and V at 23.3 clicks/sec and for wave $\mathrm{V}$ only at 77.7 clicks/sec. The low- and highfrequency filters were set at 100 and $1500 \mathrm{~Hz}$, respectively. A maximum of 2,000 sweeps was collected. The MLR was recorded using a $100-\mu$ sec alternating click stimulus at $70 \mathrm{~dB}$ nHL. Amplitudes from baseline were collected for the $\mathrm{Na}$ and $\mathrm{Pa}$ waveforms at a rate of 7.1 clicks/sec. A maximum of 1,000 sweeps was collected. The low- and high-frequency filters were set at 10 and $1500 \mathrm{~Hz}$, respectively. Both ABR and MLR testing consisted of two trials. The average of the two trials was used for analysis. For each participant, the ABR was conducted first followed by the MLR. Behavioral and electrophysiological testing were pseudorandomized between participants.

\section{RESULTS}

D articipants were separated into Pre-M and Peri/ Post-M groups. An analysis of variance (ANOVA) was performed to address the aims of the study. The ANOVAs had the independent variable menopausal status (Pre-M versus Peri/Post-M), with the dependent variables varying by analysis. The dependent variables included the following: percent correct performance on the DD, duration patterns, and speech recognition in noise tests; signal-to-noise ratio thresholds on the Listening in Spatialized Noise test; and amplitude and latency measurements made on the auditory brainstem response and MLR. Trends in the descriptive statistics were considered when significance was noted.

For duration patterns, DD, and SPIN-R, mean results are depicted in Figures 1-3, respectively. Results

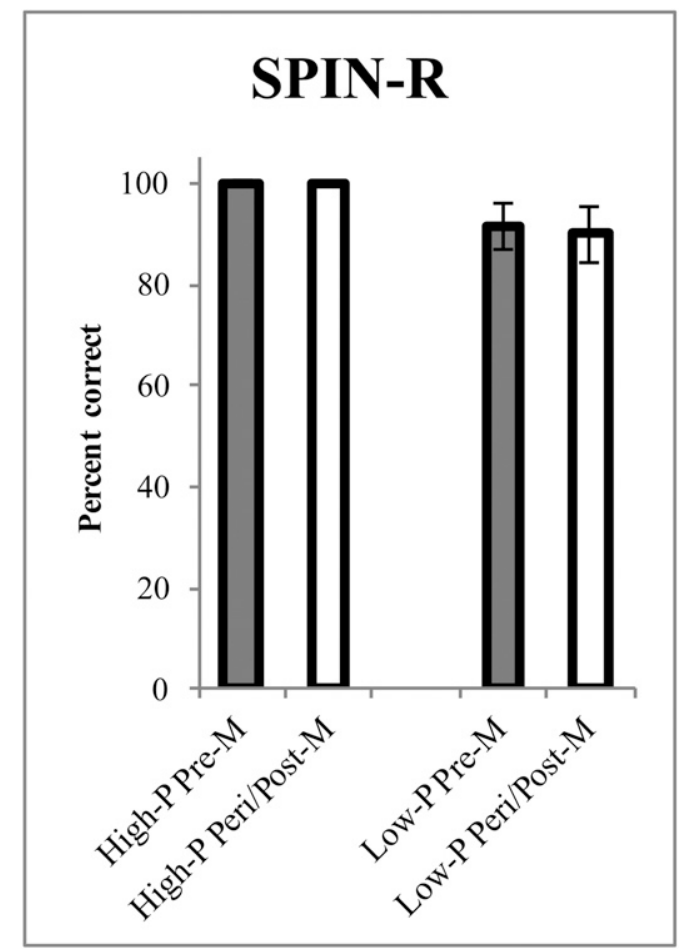

Figure 3. Means for the SPIN-R test for pre- vs. postmenopausal women comparing low vs. high predictability sentences. Error bars represent one standard deviation. Pre-M = premenopausal; Peri/Post-M = peri-/postmenopausal; High-P = high probability; Low-P = low probability. 


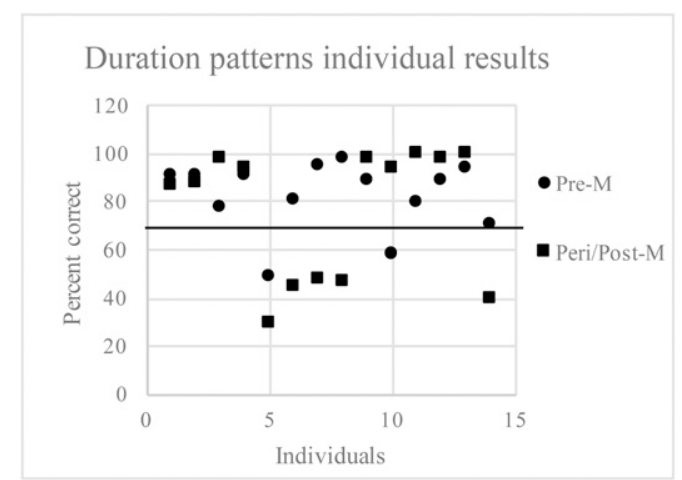

Figure 4. Individual results for the duration patterns test with threshold bars representing normative values. Pre-M = premenopausal; Peri/Post-M = peri-/postmenopausal.

for these tests are shown as mean percent correct on each individual test with error bars representing one standard deviation. DD are subdivided between right and left ears whereas SPIN-R test is subdivided into high-probability and low-probability words. In addition, Figures 4-6 show results of the behavioral testing for each individual. Scores below the threshold lines are considered failing. On ANOVA analysis, no significant differences were detected between groups in the duration patterns, DD, or SPIN-R tests.

ANOVA analysis was conducted and significant differences identified in the LiSN-S test with the Peri/ Post-M group performing more poorly in low-cue speech recognition threshold (SRT) (Pre-M $=-1.7231 \pm$ $0.77153 \mathrm{~dB}$, Peri/Post-M $=-0.9971 \pm 0.89672, p=$ 0.034), high-cue SRT (Pre-M $=-15.515 \pm 1.3868 \mathrm{~dB}$, Peri/Post-M $=-12.893 \pm 1.9105, p=0.000$ ), as well as overall performance (Pre-M $=-13.7615 \pm 1.34073 \mathrm{~dB}$, Peri/Post-M $=11.8743 \pm 1.89315, p=0.007$ ). The significant results of the LiSN-S test are demonstrated in Figure 7.

As seen in Figures 8 and 9, the two electrophysiological tests conducted were the ABR and MLR. In addi- tion, Figure 10 shows the results of the ABR for each individual with threshold bars representing normative values. On ANOVA analysis, there was a significant difference in Wave V on the left side only with the Peri/ Post-M group having a longer latency on an absolute scale (Pre-M = $3.7179 \pm 0.13239 \mathrm{~ms}$, Peri/Post-M = $3.8486 \pm 0.16640 \mathrm{~ms}, p=0.030$ ). There were also significant differences between groups in the rate effects on wave $\mathrm{V}$ bilaterally with the Peri/Post-M group having longer latencies both on the left (Pre-M $=0.3600 \pm$ $0.14676 \mathrm{~ms}$, Peri/Post-M $=0.7285 \pm 0.56657 \mathrm{~ms}, p=$ 0.027 ) and on the right (Pre-M $=0.4079 \pm 0.08613 \mathrm{~ms}$, Peri/Post-M $=0.5650 \pm 0.24951 \mathrm{~ms}, p=0.035)$. The rate effect is calculated as the latency difference of wave $\mathrm{V}$ recorded when the repetition rate is 23.3 clicks/sec versus 77.7 clicks/sec. There were otherwise no significant differences in ABR variables. Statistically significant differences were also observed for the MLR. Specifically, the ANOVA analysis demonstrated a significant difference in the right side $\mathrm{Pa}$ amplitude with the Peri/Post-M group demonstrating a larger amplitude (Pre-M $=0.5707 \pm 0.19543 \mu \mathrm{V}$, Peri/Post-M = $0.7292 \pm 0.15697 \mu \mathrm{V}, p=0.029$ ). There were otherwise no significant differences in MLR variables.

\section{DISCUSSION}

lthough there is evidence detailing the effects of feA male hormonal changes on the ABR (Jerger and Hall, 1980; Wharton and Church, 1990; Elkind-Hirsch et al 1992a,b; Caruso et al, 2000; Caruso et al, 2003; Khaliq et al, 2003; Serra et al, 2003), little research has been conducted on other measures of auditory processing in relation to female hormonal changes. The aim of this study was to compare the effects of these hormonal changes on auditory processing.

There is increasing interest in studying menopause as a risk factor for hearing loss. One prospective cohort

\section{Dichotic digits individual results}
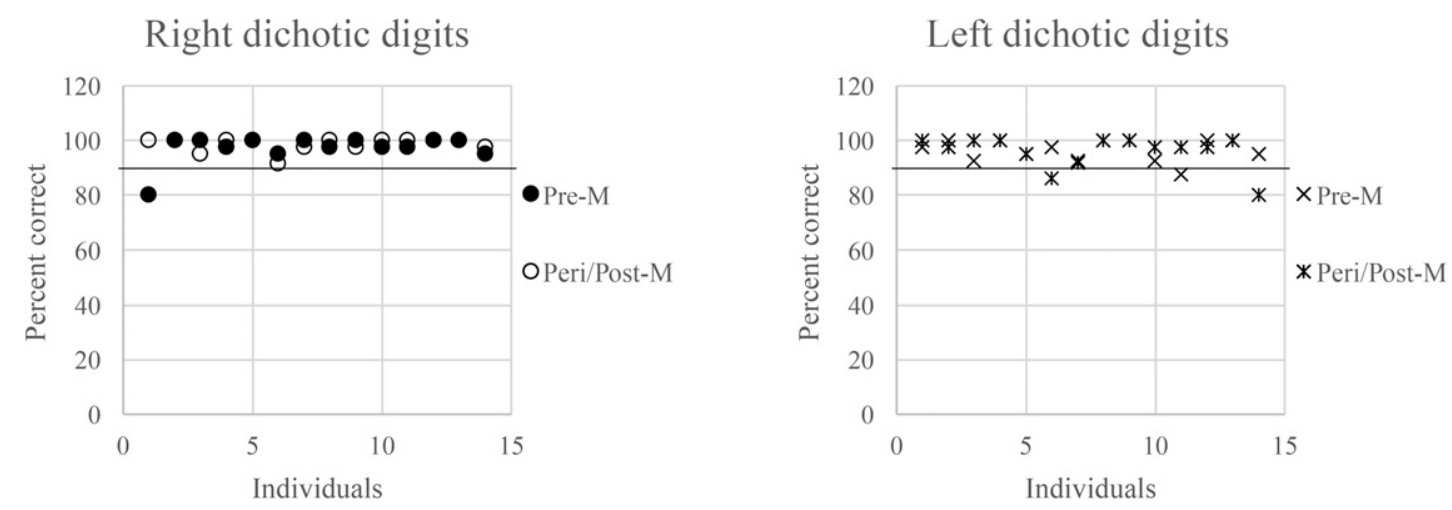

Figure 5. Individual results for the dichotic digits test with threshold bars representing normative values. Pre-M = premenopausal; Peri/Post-M = peri-/postmenopausal. 


\section{SPIN-R results}

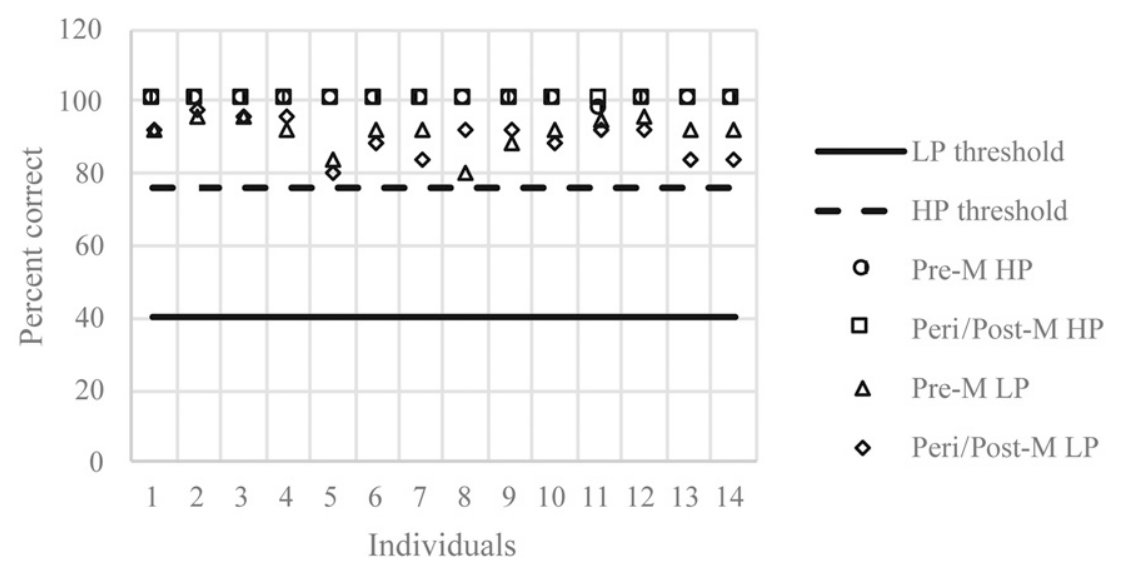

Figure 6. Individual results for the SPIN-R test with threshold bars representing normative values. Pre-M = premenopausal; Peri/Post$\mathrm{M}=$ peri-/postmenopausal; $\mathrm{HP}=$ high probability; LP = low probability.

study analyzing 80,972 women showed that although menopausal status was not associated with a higher risk of hearing loss, undergoing menopause at age $50+$ years did yield a higher risk (Curhan et al, 2017). This study found that, contrary to studies investigating the effects of HRT on the ABR, Post-M HRT was associated with an increased risk of self-reported hearing loss (Curhan et al, 2017). Hederstierna et al instead examined pure-tone audiometry as a measure of hearing loss and found that Post-M women not taking HRT had worse hearing at the $2-$ and $3-\mathrm{kHz}$ range (Hederstierna et al, 2007). Post-M women have also been found to have increased rates of hearing decline on pure-tone audiometry (Svedbrant et al, 2015) and Post-M women with decreased bone mineral density have a higher prevalence of age-related sensorineural hearing loss (Kim et al, 2016). Although the effect of HRT on hearing is controversial, hearing loss defined by self-report or pure-tone audiometry is clearly only one part of the picture in the audiological management of Post-M women. None of these studies, however, investigated central auditory processing as a possible etiology of self-reported hearing loss.

Although the traditional tests of auditory processing did not yield statistically significant differences, the Peri/Post-M group did significantly worse on the LiSN-S test, particularly on low-cue SRTs, high-cue SRT, and overall. The LiSN-S test provides objective data on the participant's ability to listen in background noise and abnormal test results may point to CAPDs (Cameron and Dillon, 2007). The results of the LiSN-S test show that the Peri/Post-M women have difficulty listening in background noise when there are neither talker and spatial differences between target sentences and background noise (low cue) and when there are both talker and spatial differences (high cue). This aligns with the primary complaint among this clinical population which is difficulty hearing in noise. All of

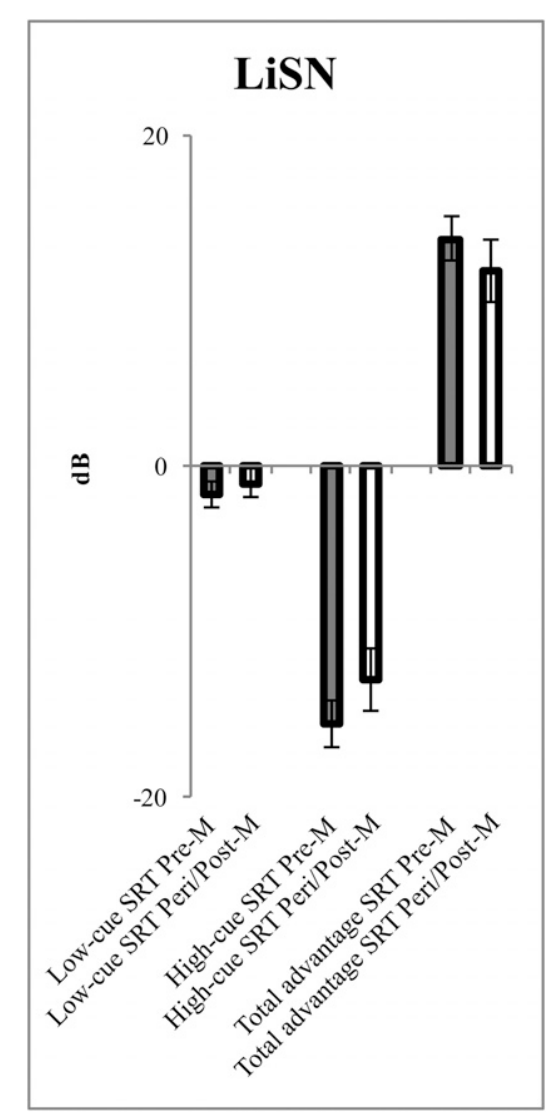

Figure 7. Means for the LiSN-S test for pre- vs. postmenopausal women comparing across the two conditions. Error bars represent one standard deviation. Pre-M = premenopausal; Peri/Post-M = peri-/postmenopausal. 


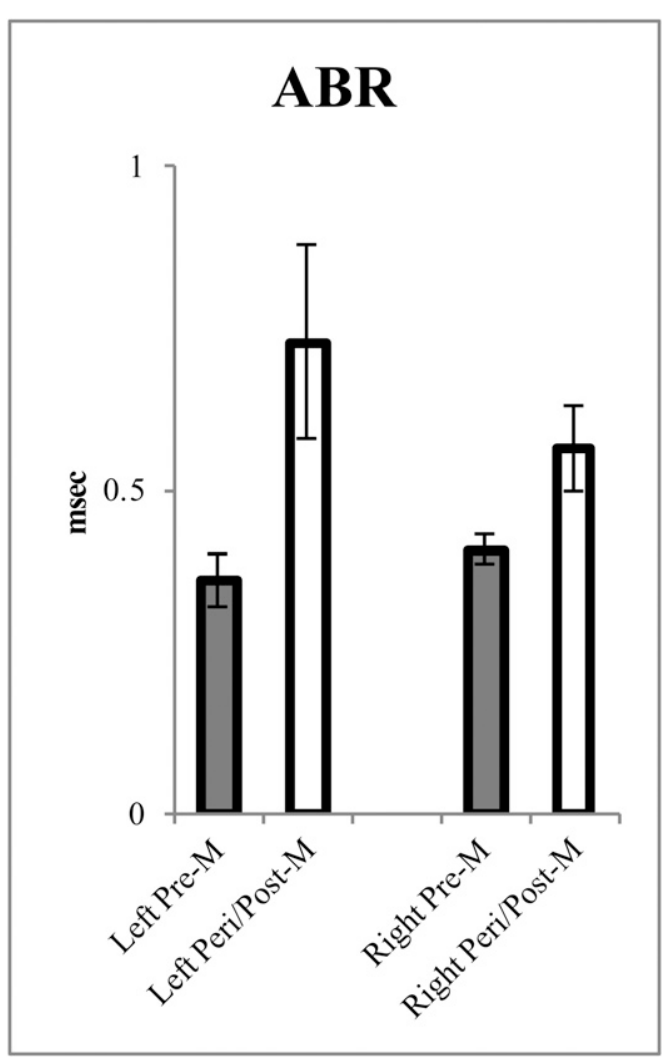

Figure 8. Means for the auditory brainstem response rate effect (wave V latency at increased stimulus rate of 77.7 clicks/sec) for pre- vs. postmenopausal women. Error bars represent one standard deviation. Pre-M = premenopausal; Peri/Post-M = peri-/ postmenopausal.

these women had normal pure-tone audiograms, therefore these difficulties hearing in noise may be attributed to possible central auditory changes. Although these findings should not be interpreted, a diagnosis of a central auditory processing deficit, it does suggest that there are central auditory differences in Pre-M versus Post-M women. Post-M women may present to an audiology clinic with a normal pure-tone audiogram and variety of auditory complaints such as difficulty hearing (especially in the presence of background noise), difficulty localizing sound, or difficulty discriminating similar sounds.

One of the more interesting findings is this study is the electrophysiological results. The ABR provides a noninvasive way to observe neural conduction in the brainstem in response to a stimulus, which is a direct look at a portion of the auditory pathways involved in auditory processing (Jewett et al, 1970). The two cohorts demonstrated similarities on the ABR, except for the effects of stimulus rate on wave $\mathrm{V}$ and the left wave III. The Peri/Post-M group had significantly longer latencies bilaterally with a higher stimulus rate.

The electrophysiological results may be explained by estrogen's role as a neuromodulator, particularly its ef-

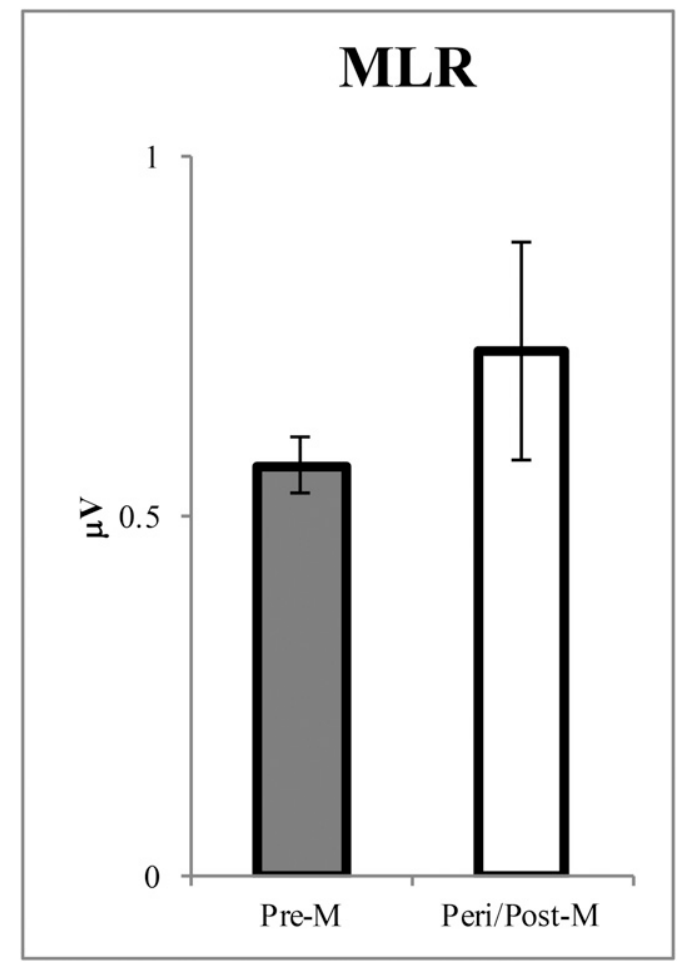

Figure 9. Means for the middle latency response - right $\mathrm{Pa}$ amplitude from baseline for pre- vs. postmenopausal women. Error bars represent one standard deviation. Pre- $\mathrm{M}=$ premenopausal; Peri/Post-M = peri-/postmenopausal.

fect on GABA-ergic neurons (Pinaud and Tremere, 2012). The increased stimulus rate causes increased synaptic firing of auditory neurons, which causes release of estrogen as it engages in postsynaptic suppression of inhibitory GABA-ergic neurons. The Peri/ Post-M group, with lower amounts of estrogen, have less estrogen-derived suppression of inhibitory GABA-ergic neurons during high stimulation and thus have more inhibition of postsynaptic neurons in the auditory pathway, which may manifest as longer latencies with higher stimulus rates. There was also a significant difference in latencies between groups on the left-sided wave III of the ABR. This may be an artifact of an underpowered study as the Peri/Post-M state is characterized by a systemic decrease in estrogen production, which should cause bilateral effects, not unilateral. A similar reasoning may account for the single significant difference found on the right-sided Pa Amplitude of the MLR. This finding certainly needs further investigation.

There is evidence in the literature to support the role of estrogen on the ABR. Elkind-Hirsch et al (1992a), evaluated how the menstrual cycle influenced the ABR. They found that the ABR is sensitive to fluctuations in serum estrogen. These authors also demonstrated increased wave $\mathrm{V}$ latencies in young women with premature ovarian failure who were undergoing estrogen and progesterone replacement therapy. They 
Auditory brainstem response individual results
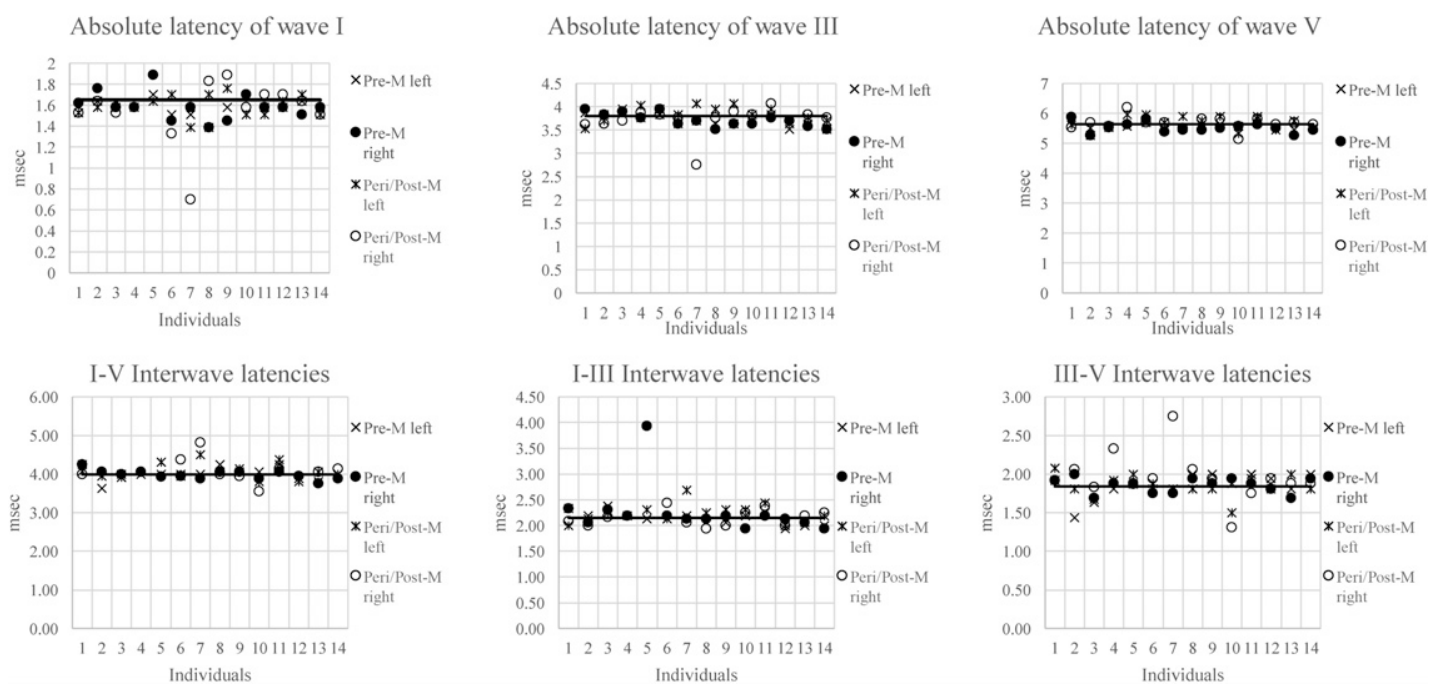

Figure 10. Individual results for the auditory brainstem response with threshold bars representing normative values. Pre-M = premenopausal; Peri/Post-M = peri-/postmenopausal.

hypothesized that synaptic conduction time may be delayed due to inhibition of the GABA synapses and that this may in fact be exacerbated by progesterone (Elkind-Hirsch et al, 1992b). The electrophysiological evidence for subtle differences in auditory function in Pre-M versus Post-M changes is an important finding and further supports the notion that we cannot rely solely on the pure-tone audiogram to detect differences or deficits in auditory processing.

There are several limitations in this study. The study protocol did not screen for any other risk factors such as hypertension, diabetes, or cholesterol levels. These have been found to have an association with hearing loss possibly due to a vascular pathogenesis (Gates et al, 1993) and although participants in this study have normal pure-tone audiograms, it does not exclude the possibility of cerebrovascular disease affecting the auditory processing pathway. However, age-related vascular changes or age-related processing dysfunction would be expected in the $6^{\text {th }}$ or $7^{\text {th }}$ decade of life; the Post-M women in our study had a mean age of 54. Thus, age-related causes of hearing problems would not be expected in our study population. Groups were classified based on participant-reported menstrual history, which acts as a surrogate for true estrogen levels.

\section{CONCLUSION}

$\mathrm{T}$ here is some degree of debate regarding "hearing loss" in Post-M women. What one must consider is how "hearing loss" is defined. Although it may be the case that Post-M women are not at risk for peripheral hearing loss of sensitivity, it does not rule-out a
CAPD secondary to hormonal changes. Significant differences in auditory processing tests exist between Pre-M and Peri/Post-M women. This includes listening in noise and the effect of stimulus rate on wave $V$ of the auditory brainstem response. Post-M women presenting with auditory complaints but normal hearing thresholds warrant further audiological evaluation for possible deficits in central auditory processing, a clinical problem that may otherwise go unrecognized.

\section{REFERENCES}

Bilger RC, Nuetzel JM, Rabinowitz WM, Rzeczkowski C. (1984) Standardization of a test of speech perception in noise. $J$ Speech Hear Res 27:32-48.

Cameron S, Dillon H. (2007) Development of the listening in spatialized noise-sentences test (LISN-S). Ear Hear 28:196-211.

Caruso S, Cianci A, Grasso D, Agnello C, Galvani F, Maiolino L, Serra A. (2000) Auditory brainstem response in postmenopausal women treated with hormone replacement therapy: a pilot study. Menopause 7:178-183.

Caruso S, Maiolino L, Agnello C, Garozzo A, Di Mari L, Serra A. (2003) Effects of patch or gel estrogen therapies on auditory brainstem response in surgically postmenopausal women: a prospective, randomized study. Fertil Steril 79:556-561.

Coleman JR, Campbell D, Cooper WA, Welsh MG, Moyer J. (1994) Auditory brainstem responses after ovariectomy and estrogen replacement in rat. Hear Res 80:209-215.

Curhan SG, Eliassen AH, Eavey RD, Wang M, Lin BM, Curhan GC. (2017) Menopause and postmenopausal hormone therapy and risk of hearing loss. Menopause 24:1049-1056.

Elkind-Hirsch KE, Stoner WR, Stach BA, Jerger JF. (1992a) Estrogen influences auditory brainstem responses during the normal menstrual cycle. Hear Res 60:143-148. 
Elkind-Hirsch KE, Stoner WR, Stach BA, Jerger JF. (1992b) Cyclic steroid replacement alters auditory brainstem responses in young women with premature ovarian failure. Hear Res 64:93-98.

Gates GA, Cobb J, D'Agostino RB, Wolf PA. (1993) The relation of hearing in the elderly to the presence of cardiovascular disease and cardiovascular risk factors. Arch Otolaryngol Head Neck Surg 119:156-161.

Golub MS, Germann SL, Hogrefe CE. (2004) Endocrine disruption and cognitive function in adolescent female rhesus monkeys. Neurotoxicol Teratol 26:799-809.

Hederstierna C, Hultcrantz M, Collins A, Rosenhall U. (2007) Hearing in women at menopause. Prevalence of hearing loss, audiometric configuration and relation to hormone replacement therapy. Acta Otolaryngol 127:149-55.

Hultcrantz M, Sylvén L. (1997) Turner's syndrome and hearing disorders in women aged 16-34. Hear Res 103:69-74.

Hultcrantz M, Simonoska R, Stenberg AE. (2006) Estrogen and hearing: a summary of recent investigations. Acta Otolaryngol 126:10-14.

Jerger J, Hall J. (1980) Effects of age and sex on auditory brainstem response. Arch Otolaryngol 106:387-391.

Jewett DL, Romano MN, Williston JS. (1970) Human auditory evoked potentials: possible brain stem components detected on the scalp. Science 167:1517-1518.

Khaliq F, Tandon OP, Goel N. (2003) Auditory evoked responses in postmenopausal women on hormone replacement therapy. Indian J Physiol Pharmacol 47:393-399.

Kilicdag EB, Yavuz H, Bagis T, Tarim E, Erkan AN, Kazanci F. (2004) Effects of estrogen therapy on hearing in postmenopausal women. Am J Obstet Gynecol 190:77-82.

Kim JY, Lee SB, Lee CH, Kim HM. (2016) Hearing loss in postmenopausal women with low bone mineral density. Auris Nasus Larynx 43:155-60.

Laugel GR, Dengerink HA, Wright JW. (1987) Ovarian steroid and vasoconstrictor effects on cochlear blood flow. Hear Res 31:245-251.

Lin FR, Niparko JK, Ferrucci L. (2011) Hearing loss prevalence in the United States. Arch Intern Med 171:1851-1852.
Maney DL, Cho E, Goode CT. (2006) Estrogen-dependent selectivity of genomic responses to birdsong. Eur J Neurosci 23:1523-1529.

Mulrow CD, Aguilar C, Endicott JE, Tuley MR, Velez R, Charlip WS, Rhodes MC, Hill JA, DeNino LA. (1990a) Qualityof-life changes and hearing impairment. A randomized trial. Ann Intern Med 113:188-194.

Mulrow CD, Aguilar C, Endicott JE, Velez R, Tuley MR, Charlip WS, Hill J. (1990b) Association between hearing impairment and the quality of life of elderly individuals. J Am Geriatr Soc $38: 45-50$.

Musiek FE. (1983) Assessment of central auditory dysfunction: the dichotic digit test revisited. Ear Hear 4:79-83.

Musiek FE, Baran JA, Pinheiro ML. (1990) Duration pattern recognition in normal subjects and participants with cerebral and cochlear lesions. Audiology 29:304-13.

Pinaud R, Tremere LA. (2012) Control of central auditory processing by a brain-generated oestrogen. Nat Rev Neurosci 13:521-527.

Serra A, Maiolino L, Agnello C, Messina A, Caruso S. (2003) Auditory brain stem response throughout the menstrual cycle. Ann Otol Rhinol Laryngol 112:549-553.

Shinn J, Long A, Rayle C, Bush M. (2016) Primary auditory symptoms in participants with normal peripheral hearing sensitivity: redefining hearing loss. Hearing, Balance Commun 14: 44-49.

Stenberg AE, Wang H, Fish J, 3rd, Schrott-Fischer A, Sahlin L, Hultcrantz M. (2001) Estrogen receptors in the normal adult and developing human inner ear and in Turner's syndrome. Hear Res 157:87-92.

Stenberg AE, Wang H, Sahlin L, Hultcrantz M. (1999) Mapping of estrogen receptors alpha and beta in the inner ear of mouse and rat. Hear Res 136:29-34.

Svedbrant J, Bark R, Hultcrantz M, Hederstierna C. (2015) Hearing decline in menopausal women-a 10-year follow-up. Acta Otolaryngol 135:807-813.

Wharton JA, Church GT. (1990) Influence of menopause on the auditory brainstem response. Audiology 29:196-201. 